\title{
Data Consistency Tests through the Use of Neural Networks and Virial Equation. Application of the Proposed Methodology to Critical Study of Density Data
}

\author{
Serge Laugier ${ }^{*}$, Hakim Madani ${ }^{2}$, Abdeslam Hassen Meniai ${ }^{3}$, Dominique Richon ${ }^{4}$ \\ ${ }^{1}$ I2m, Umr Cnrs 5295 Enscbp, Pessac, France \\ ${ }^{2}$ Laboratoire d'études des Systèmes Energétiques Industriels, Université de Batna, Batna, Algeria \\ ${ }^{3}$ Laboratoire de l'Ingénierie des Procédés d'Environnement, Université Mentouri Constantine, Constantine, Algeria \\ ${ }^{4}$ MINES ParisTech, Centre Énergétique et Procédés, 35 Rue Saint Honoré, Fontainebleau, France \\ *E-mail: laugier@enscbp.fr \\ Received May 30, 2011; revised August 9, 2011; accepted September 11, 2011
}

\begin{abstract}
This paper focuses on a very important point which consists in evaluating experimental data prior to their use for chemical process designs. Hexafluoropropylene $P, \rho, T$ data measured at 11 temperatures from 263 to $362 \mathrm{~K}$ and at pressures up to $10 \mathrm{MPa}$ have been examined through a consistency test presented herein and based on the use of a methodology implying both neural networks and Virial equation. Such a methodology appears as very powerful to identify erroneous data and could be conveniently handled for quick checks of databases previously to modeling through classical thermodynamic models and equations of state. As an application to liquid and vapor phase densities of hexafluoropropylene, a more reliable database is provided after removing out layer data.
\end{abstract}

Keywords: Consistency Tests, Hexafluoropropylene, Neural Networks, Vibrating Tube Densimeter, Virial Equation

\section{Introduction}

Nowadays a great concern towards the environment protection is shown by the refrigeration industry which is urged to find new fluids as refrigerants substitutes. In Montreal Protocol (1987) it was decided to phase out and replace ozone-depleting refrigerants like chlorofluorocarbons (CFC's) and hydrochlorofluorocarbons (HCFC's). Consequently CFCs were prohibited in 1996 by the signing of Montreal Protocol countries and the fixed deadline for a total banishment of HCFC's which have low ozone depletion potential was set, i.e. 2030 [1].

Alternative compounds must be found and hexafluoropropene (HFP, R1216), CAS Number 116-15-4 is good candidate with its 0.86 GWP value [2], eventually mixed to other components. Numerous data concerning volumetric properties of this compound obtained using the vibrating-tube densitometer technique are available in Coquelet et al. paper [3]. They correspond to $P, \rho, T$ triplets belonging to 11 isotherms reported partly in tables 2 and 3. It is highly recommended prior to using data for process design to have serious estimation about their reliability and their accuracy, mainly in order not to take too big error margins. The best recommendation that could be done to experimental laboratories would be the use of several experimental techniques to check for reproducibility of data [4] and consequently provide guaranteed data. Young researchers must be encouraged to handle experimental works that are so useful to industry and theoricists [5]. Collaborations between laboratories with complementary skills in either experimental, modeling and simulation are a real advantage for presenting worth and reliable data. Starting a French thermodynamic research federation under the auspices of CNRS is in project along with the setup of an international network. The following sentence [6]: "All of the just mentioned points need to be addressed in the frame of a thermodynamicists' network. This is one of the urgent goals to be achieved in the near future" is found just after a list of comments and remarks done during round table discussions.

For low pressure $P V T$ data, a very simple test consists in verifying the data do follow the virial equation and agree with the perfect gas law when pressure tends to 
zero (density tends to zero while pressure tends to zero).

For high pressure PVT data, the use of a flexible model, as a neural network based model, allows testing internal constancy of data.

These approaches are enlightened in this paper. They are complementary tests to over, for example see references [7-9], but are the most convenient here, in the considered pressure range.

\section{Thermodynamic Consistency of Data in Vapor Phase through the Virial Equation}

At low pressures the use of the virial equation is quite convenient to check for data consistency with respect to vapor phase. Indeed, it is well known that the virial equation, truncated after the 3rd term allows acceptable representation of PVT data of pure compounds at low and medium pressures (up to about $2 \mathrm{MPa}$ ).

This equation is written as:

$$
\text { P.v/R.T }=1+B / v+C / v^{2}
$$

with $B$ and $C$ being the 2nd and 3rd virial coefficients.

These two parameters can be adjusted from isothermal experimental data by rewriting Equation (1) as:

$$
\text { (P.v/R.T-1). } v=B+C / v
$$

By tracing the evolution of the $((P . v / R . T)-1) . v$ term versus $1 / v$, it is possible to check for the linearity of Equation (2). Applied to data at $348.1 \mathrm{~K}$, the results are shown in Figure 1. Perfect linearity is observed for $1 / \mathrm{V}$ above $0.4 \mathrm{kmol} \cdot \mathrm{m}^{-3}$ i.e. above $1.0 \mathrm{MPa}$. But, at lower pressures, the experimental points depart significantly from linearity. Experimental uncertainties cannot explain these discrepancies while the virial equation is all the more justified for the lowest pressures.

Such departures, at low pressures, displayed at 348.1 $\mathrm{K}$ are observed for all of the other temperatures. This observation proves that although the densimeter vibrating tube [3] is ideal for measuring medium or high density values it is no longer the ideal tool for very low densities.

Although a low pressure "pressure transducer" was used, when working at the lowest pressures, this was not the solution for ensuring quality density values. It is reasonable to believe that densimeter designed for working at pressures up to $40 \mathrm{MPa}$, is not convenient at low pressures because of its thick-walled (not sensitive enough) vibrating tube.

For low pressures, the values of data P.V.T can be extrapolated preferably and conveniently from the data obtained at higher pressures, by using the virial equation. In Table 1, are given the values of the 2nd and 3rd virial coefficients calculated from the reliable part of the data.

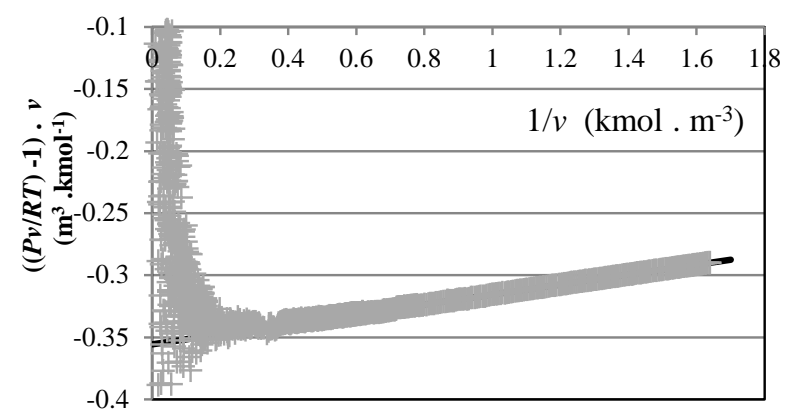

Figure 1. Data departure from virial equation at 348.1 K.

Table 1. 2nd and 3rd virial coefficients as a function of temperature.

\begin{tabular}{ccc}
\hline$T$ & $B$ & $C$ \\
\hline $\mathrm{K}$ & $\mathrm{m}^{3} \cdot \mathrm{kmol}^{-1}$ & $\mathrm{~m}^{6} \cdot \mathrm{kmol}^{-2}$ \\
283.24 & -0.5400 & -0.17000 \\
303.28 & -0.4880 & -0.01124 \\
323.21 & -0.4283 & 0.04116 \\
343.26 & -0.3720 & 0.04303 \\
348.12 & -0.3556 & 0.04015 \\
353.12 & -0.3432 & 0.03953 \\
355.27 & -0.3384 & 0.03920 \\
357.06 & -0.3344 & 0.03912 \\
358.16 & -0.3325 & 0.03903 \\
362.90 & -0.3201 & 0.03743 \\
\hline
\end{tabular}

\section{Thermodynamic Consistency of Data in Liquid Phase through a Multiparameter Model}

A multiparameter model allows the representation of experimental data within their experimental uncertainties, provided the uses of a sufficient number of parameters and the availability of great enough number of data.

When a reduced number of data are not consistent with respect to main part of database, the model will lead to a representation presenting deviations much higher than estimated values of experimental uncertainties. Consequently, doubtful data will be easily identified through deviation plots.

Multiparameter models must have certain characteristics: they must be very flexible and able to admit a great number of parameters. A neural network based model has all these characteristics. It will be used in this work. Detailed description of neural network models is given elsewhere [10,11]. Figure 2 shows the topology of a neural network with only one hidden layer. The activation function, used herein, is the sigmoid one. Through optimization procedure the adequate number of neurons in hidden layer was found to be 7 , allowing both under and over-fittings. The two neurons in the input layer represent the following independent variables: temperature $\mathrm{T}$ and density $\rho$, while the output variable is the pressure P. All variables are normalized between 0.1 and 0.9 to deal with 


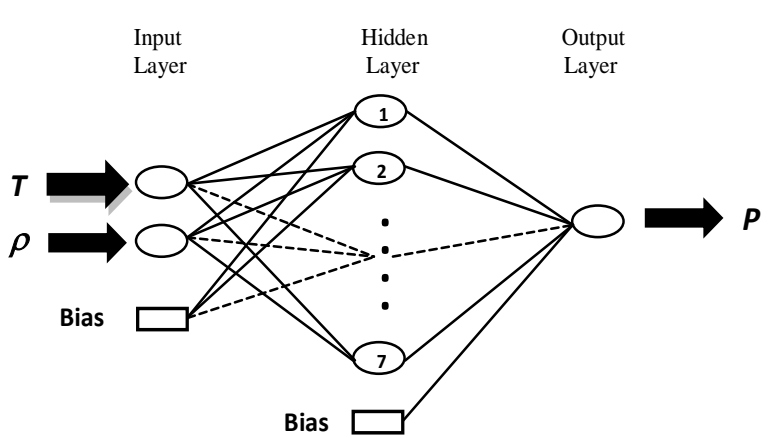

Figure 2. Neural network topology.

large variable ranges.

We have selected 4300 triplets in a random way among not the published data 3 but the raw data as they were got by the authors through their data acquisition unit (about 48,000 ( $P, \rho, T)$ triplets), for the training the network (adjustment of its 29 parameters). This permits observing the deviations between experimental and calculated pressures for these 48,000 triplets. It is worth to use raw data instead of published data as they are not rounded and consequently have more digits available for mathematical treatments. Special behavior is pointed out at $348.2 \mathrm{~K}$, see Figure 3. In fact, between 8.5 and $10 \mathrm{MPa}$, several points evolve in a way different from most of the others. To enlight clearly this problem, we have plotted (see Figure 4) the density as a function of pressure in the (8-10) MPa range. One observes two distinct series of data. The series corresponding to the lowest density values but also to the highest Pexp-Pcal deviations must be eliminated from the corresponding isothermal set data (30 experimental points). A new neural network treatment on the $348 \mathrm{~K}$ isothermal data after removing doubtful data was carried out adjusting again 29 parameters. Even after discarding these 30 experimental points too high deviations are still observed; they concern 450 data that must be also discarded. Four successive treatments have been necessary to obtain a clean coherent dataset (deviations in agreement with estimated experimental uncertainties).

Behaviors similar to those displayed in Figures $\mathbf{3}$ and $\mathbf{4}$ are found at all temperatures. Corresponding complementary figures are available upon request to the corresponding author of this paper. All isothermal data at 355.2 $\mathrm{K}$ must be rejected, the reason of rejection being the high standard deviation that is observed between experimental and calculated pressures; It is, in fact, 5 times bigger for this isotherm than for all other isotherms. Finally, 42,240 triplets are considered as trustable over the 48,000. Pressure deviations corresponding to these 42,240 triplets are plotted in Figure 5. They are contained in $+/-0.04 \mathrm{MPa}$ pressure range, although authors [3] claimed for pressure uncertainties within 0.0003 or $0.0006 \mathrm{MPa}$, depending on

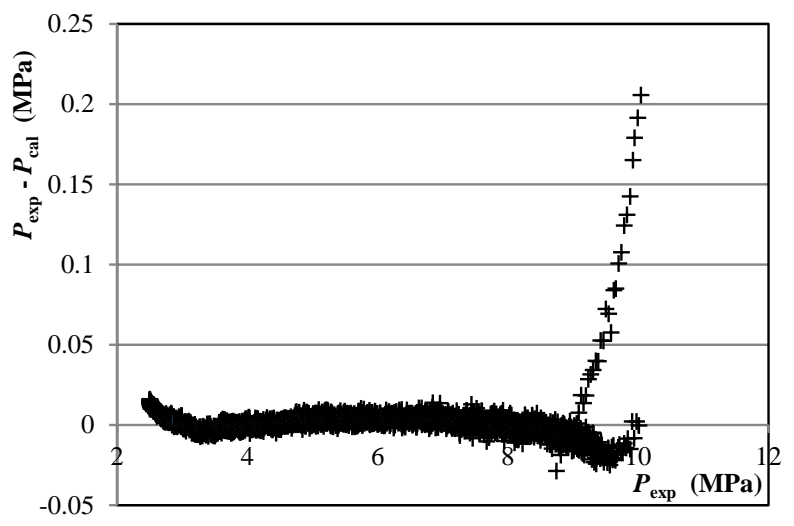

Figure 3. Deviations between experimental and calculated pressures at $348.2 \mathrm{~K}$.

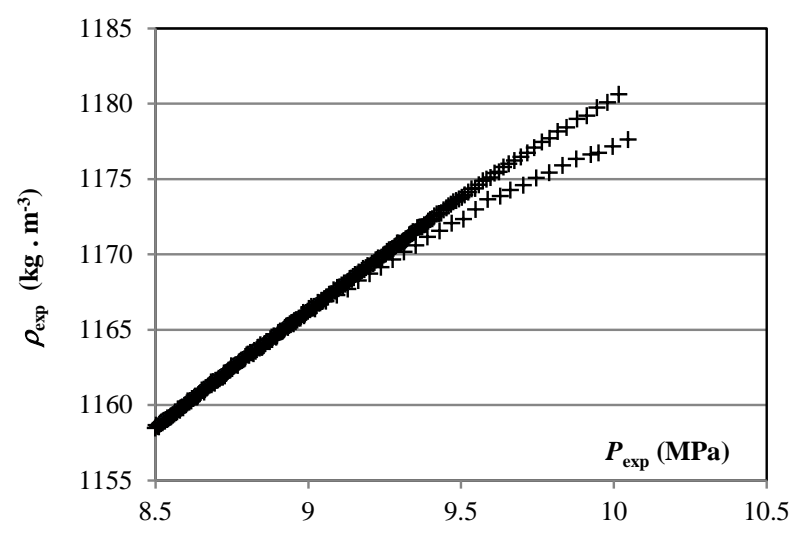

Figure 4. Density as a function of pressure at $348.2 \mathrm{~K}$.

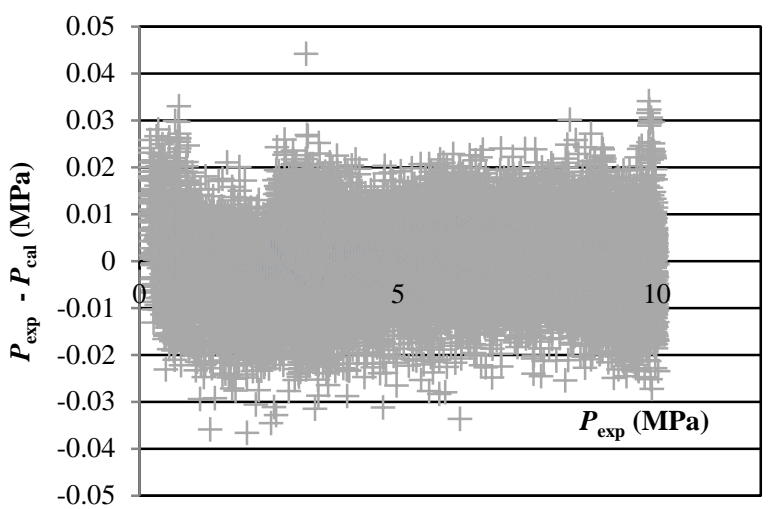

Figure 5. Deviations between experimental and calculated pressures for remaining liquid densities after consistency test treatment.

pressure sensor used. The standard deviation on the 42,240 points is $0.005 \mathrm{MPa}$, this is about ten times larger than their estimated pressure uncertainties. We can conclude uncertainties on pressures were evidently underestimated by Coquelet et al. [3].

Table 2 contains only the trustable data from Tables 2 
Table 1. Densities of HFP.

\begin{tabular}{|c|c|c|c|c|c|c|c|c|c|c|c|}
\hline \multicolumn{12}{|c|}{ Vapor densities } \\
\hline$T$ & $P$ & $\rho$ & $T$ & $P$ & $\rho$ & $T$ & $P$ & $\rho$ & $T$ & $P$ & $\rho$ \\
\hline K & $\mathrm{MPa}$ & $\mathrm{kg} \cdot \mathrm{m}^{-3}$ & $\mathrm{~K}$ & $\mathrm{MPa}$ & $\mathrm{kg} \cdot \mathrm{m}^{-3}$ & $\mathrm{~K}$ & $\mathrm{MPa}$ & $\mathrm{kg} \cdot \mathrm{m}^{-3}$ & $\mathrm{~K}$ & $\mathrm{MPa}$ & $\mathrm{kg} \cdot \mathrm{m}^{-3}$ \\
\hline 263.41 & 0.1302 & 9.2809 & 263.40 & 0.2274 & 16.933 & 283.24 & 0.4445 & 32.350 & 303.28 & 0.7729 & 56.178 \\
\hline 263.41 & 0.1330 & 9.5029 & 263.40 & 0.2294 & 17.053 & 283.24 & 0.4483 & 32.640 & 303.28 & 0.7821 & 57.024 \\
\hline 263.40 & 0.1358 & 9.7287 & 263.41 & 0.2314 & 17.292 & 283.24 & 0.4521 & 32.992 & 303.28 & 0.7912 & 57.852 \\
\hline 263.40 & 0.1386 & 9.9512 & & & & 283.25 & 0.4559 & 33.232 & 303.28 & 0.8003 & 58.744 \\
\hline 263.41 & 0.1415 & 10.136 & 283.23 & 0.2895 & 19.903 & 283.25 & 0.4605 & 33.768 & 303.28 & 0.8105 & 59.713 \\
\hline 263.41 & 0.1442 & 10.379 & 283.24 & 0.2953 & 20.300 & & & & 303.26 & 0.8187 & 60.522 \\
\hline 263.41 & 0.1471 & 10.581 & 283.24 & 0.3035 & 20.988 & 303.28 & 0.4206 & 27.450 & 303.27 & 0.8270 & 61.310 \\
\hline 263.40 & 0.1498 & 10.801 & 283.24 & 0.3084 & 21.365 & 303.27 & 0.4316 & 28.275 & 303.28 & 0.8340 & 62.058 \\
\hline 263.40 & 0.1525 & 10.973 & 283.23 & 0.3133 & 21.707 & 303.28 & 0.4439 & 29.151 & 303.28 & 0.8420 & 62.873 \\
\hline 263.40 & 0.1553 & 11.212 & 283.24 & 0.3174 & 22.036 & 303.28 & 0.4548 & 29.939 & & & \\
\hline 263.40 & 0.1579 & 11.414 & 283.24 & 0.3222 & 22.368 & 303.28 & 0.4657 & 30.696 & 323.21 & 0.5008 & 30.574 \\
\hline 263.40 & 0.1606 & 11.647 & 283.24 & 0.3270 & 22.770 & 303.28 & 0.4763 & 31.544 & 323.20 & 0.5407 & 33.232 \\
\hline 263.40 & 0.1632 & 11.833 & 283.24 & 0.3312 & 23.117 & 303.28 & 0.4884 & 32.458 & 323.21 & 0.5686 & 35.175 \\
\hline 263.41 & 0.1659 & 12.035 & 283.24 & 0.3352 & 23.372 & 303.28 & 0.5004 & 33.397 & 323.20 & 0.6033 & 37.627 \\
\hline 263.41 & 0.1686 & 12.218 & 283.24 & 0.3400 & 23.845 & 303.27 & 0.5136 & 34.318 & 323.20 & 0.6395 & 40.168 \\
\hline 263.41 & 0.1712 & 12.456 & 283.24 & 0.3442 & 24.108 & 303.28 & 0.5241 & 35.196 & 323.21 & 0.6750 & 42.784 \\
\hline 263.41 & 0.1738 & 12.654 & 283.24 & 0.3482 & 24.411 & 303.28 & 0.5345 & 35.971 & 323.20 & 0.7098 & 45.300 \\
\hline 263.41 & 0.1764 & 12.818 & 283.25 & 0.3531 & 24.764 & 303.26 & 0.5450 & 36.847 & 323.21 & 0.7373 & 47.337 \\
\hline 263.40 & 0.1789 & 13.078 & 283.24 & 0.3571 & 25.072 & 303.27 & 0.5555 & 37.603 & 323.20 & 0.7846 & 50.994 \\
\hline 263.40 & 0.1815 & 13.237 & 283.25 & 0.3611 & 25.445 & 303.28 & 0.5658 & 38.412 & 323.21 & 0.8254 & 54.173 \\
\hline 263.41 & 0.1839 & 13.463 & 283.24 & 0.3659 & 25.853 & 303.28 & 0.5760 & 39.225 & 323.21 & 0.8558 & 56.561 \\
\hline 263.41 & 0.1864 & 13.619 & 283.24 & 0.3700 & 26.146 & 303.28 & 0.5875 & 40.136 & 323.21 & 0.8825 & 58.745 \\
\hline 263.41 & 0.1890 & 13.817 & 283.24 & 0.3747 & 26.552 & 303.28 & 0.5977 & 41.017 & 323.21 & 0.9164 & 61.512 \\
\hline 263.41 & 0.1913 & 14.012 & 283.24 & 0.3787 & 26.899 & 303.28 & 0.6079 & 41.804 & 323.20 & 0.9493 & 64.319 \\
\hline 263.40 & 0.1938 & 14.200 & 283.24 & 0.3828 & 27.236 & 303.28 & 0.6206 & 42.906 & 323.21 & 0.9832 & 67.213 \\
\hline 263.40 & 0.1961 & 14.396 & 283.24 & 0.3867 & 27.563 & 303.27 & 0.6306 & 43.671 & 323.20 & 1.0027 & 69.010 \\
\hline 263.40 & 0.1985 & 14.629 & 283.25 & 0.3907 & 27.782 & 303.26 & 0.6431 & 44.725 & 323.21 & 1.0261 & 71.062 \\
\hline 263.41 & 0.2009 & 14.793 & 283.24 & 0.3946 & 28.179 & 303.26 & 0.6532 & 45.583 & 323.21 & 1.0477 & 72.912 \\
\hline 263.40 & 0.2032 & 14.983 & 283.24 & 0.3987 & 28.482 & 303.27 & 0.6630 & 46.436 & 323.21 & 1.0673 & 74.738 \\
\hline 263.40 & 0.2055 & 15.135 & 283.24 & 0.4034 & 28.816 & 303.28 & 0.6730 & 47.215 & 323.20 & 1.0834 & 76.287 \\
\hline 263.40 & 0.2078 & 15.384 & 283.24 & 0.4074 & 29.204 & 303.28 & 0.6827 & 48.129 & 323.21 & 1.1048 & 78.288 \\
\hline 263.40 & 0.2099 & 15.521 & 283.24 & 0.4114 & 29.532 & 303.28 & 0.6936 & 49.011 & 323.20 & 1.1256 & 80.357 \\
\hline 263.40 & 0.2123 & 15.707 & 283.25 & 0.4162 & 29.928 & 303.27 & 0.7033 & 49.884 & 323.21 & 1.1401 & 81.784 \\
\hline 263.40 & 0.2146 & 15.892 & 283.24 & 0.4209 & 30.373 & 303.27 & 0.7129 & 50.724 & 323.20 & 1.1552 & 83.245 \\
\hline 263.41 & 0.2167 & 16.078 & 283.24 & 0.4248 & 30.649 & 303.28 & 0.7237 & 51.658 & 323.21 & 1.1675 & 84.433 \\
\hline 263.40 & 0.2188 & 16.238 & 283.24 & 0.4287 & 31.045 & 303.28 & 0.7344 & 52.626 & 323.21 & 1.1932 & 87.022 \\
\hline 263.40 & 0.2211 & 16.431 & 283.25 & 0.4327 & 31.286 & 303.28 & 0.7450 & 53.664 & 323.20 & 1.2070 & 88.441 \\
\hline 263.40 & 0.2232 & 16.590 & 283.24 & 0.4366 & 31.702 & 303.27 & 0.7544 & 54.477 & 323.20 & 1.2204 & 89.889 \\
\hline
\end{tabular}




\begin{tabular}{|c|c|c|c|c|c|c|c|c|c|c|c|}
\hline 263.40 & 0.2254 & 16.763 & 283.24 & 0.4405 & 31.968 & 303.27 & 0.7637 & 55.342 & 323.22 & 1.2326 & 91.079 \\
\hline$T$ & $P$ & $\rho$ & $T$ & $P$ & $\rho$ & $T$ & $P$ & $\rho$ & $T$ & $P$ & $\rho$ \\
\hline $\mathrm{K}$ & MPa & $\mathrm{kg} \cdot \mathrm{m}^{-3}$ & K & $\mathrm{MPa}$ & $\mathrm{kg} \cdot \mathrm{m}^{-3}$ & K & $\mathrm{MPa}$ & $\mathrm{kg} \cdot \mathrm{m}^{-3}$ & $\mathrm{~K}$ & $\mathrm{MPa}$ & $\mathrm{kg} \cdot \mathrm{m}^{-3}$ \\
\hline 323.21 & 1.2442 & 92.409 & 343.23 & 2.1308 & 184.26 & 353.13 & 2.7613 & 297.64 & 355.25 & 2.8628 & 314.75 \\
\hline 323.20 & 1.2549 & 93.544 & 343.22 & 2.1433 & 186.60 & 353.13 & 2.7695 & 302.00 & 355.25 & 2.8754 & 322.19 \\
\hline 323.20 & 1.2762 & 95.862 & 343.24 & 2.1553 & 188.96 & 353.13 & 2.7781 & 307.16 & 355.25 & 2.8897 & 331.26 \\
\hline 323.21 & 1.2944 & 97.960 & 343.25 & 2.1668 & 191.26 & 353.13 & 2.7816 & 309.46 & 355.25 & 2.9013 & 340.48 \\
\hline 323.20 & 1.3116 & 99.800 & 343.25 & 2.1791 & 193.71 & & & & & & \\
\hline 323.20 & 1.3280 & 101.85 & 343.21 & 2.1901 & 196.25 & 355.25 & 1.5818 & 101.98 & 357.02 & 1.6048 & 102.77 \\
\hline 323.20 & 1.3436 & 103.60 & 343.25 & 2.2014 & 198.59 & 355.25 & 1.6143 & 104.74 & 357.02 & 1.6737 & 108.67 \\
\hline \multirow[t]{2}{*}{323.21} & 1.3567 & 105.23 & 343.21 & 2.2117 & 200.97 & 355.25 & 1.6455 & 107.51 & 357.01 & 1.7418 & 114.76 \\
\hline & & & & & & 355.26 & 1.6750 & 110.13 & 357.02 & 1.8085 & 120.92 \\
\hline 343.24 & 1.5744 & 110.45 & 353.12 & 1.9032 & 134.30 & 355.25 & 1.7016 & 112.53 & 357.02 & 1.8630 & 126.06 \\
\hline 343.24 & 1.5997 & 113.00 & 353.13 & 1.9573 & 140.03 & 355.25 & 1.7255 & 114.77 & 357.02 & 1.9167 & 131.37 \\
\hline 343.25 & 1.6236 & 115.44 & 353.12 & 2.0110 & 146.07 & 355.25 & 1.7498 & 116.98 & 357.03 & 1.9716 & 137.03 \\
\hline 343.23 & 1.6467 & 117.95 & 353.13 & 2.0621 & 152.10 & 355.25 & 1.7730 & 119.17 & 357.04 & 2.0258 & 142.64 \\
\hline 343.24 & 1.6692 & 120.33 & 353.13 & 2.1096 & 157.94 & 355.25 & 1.7961 & 121.37 & 357.04 & 2.0760 & 148.19 \\
\hline 343.25 & 1.6910 & 122.68 & 353.13 & 2.1541 & 163.58 & 355.26 & 1.8170 & 123.34 & 357.04 & 2.1232 & 153.54 \\
\hline 343.25 & 1.7127 & 125.08 & 353.13 & 2.1957 & 169.10 & 355.25 & 1.8369 & 125.28 & 357.04 & 2.1646 & 158.38 \\
\hline 343.25 & 1.7340 & 127.50 & 353.13 & 2.2439 & 175.75 & 355.25 & 1.8567 & 127.27 & 357.04 & 2.2085 & 163.72 \\
\hline 343.25 & 1.7549 & 129.82 & 353.13 & 2.2824 & 181.43 & 355.25 & 1.9085 & 132.51 & 357.04 & 2.2521 & 169.16 \\
\hline 343.25 & 1.7746 & 132.14 & 353.13 & 2.3162 & 186.58 & 355.26 & 1.9678 & 138.66 & 357.04 & 2.2920 & 174.38 \\
\hline 343.23 & 1.7948 & 134.67 & 353.14 & 2.3510 & 192.05 & 355.26 & 2.0205 & 144.41 & 357.03 & 2.3295 & 179.35 \\
\hline 343.24 & 1.8160 & 137.14 & 353.14 & 2.3839 & 197.48 & 355.25 & 2.0652 & 149.45 & 357.03 & 2.3675 & 184.68 \\
\hline 343.24 & 1.8338 & 139.44 & 353.14 & 2.4158 & 202.92 & 355.25 & 2.1148 & 155.31 & 357.02 & 2.4042 & 190.03 \\
\hline 343.25 & 1.8513 & 141.49 & 353.14 & 2.4435 & 208.12 & 355.25 & 2.1600 & 160.70 & 357.02 & 2.4384 & 195.20 \\
\hline 343.20 & 1.8694 & 143.90 & 353.14 & 2.4703 & 213.14 & 355.24 & 2.2060 & 166.59 & 357.03 & 2.4724 & 200.51 \\
\hline 343.23 & 1.8871 & 146.21 & 353.13 & 2.4969 & 218.39 & 355.24 & 2.2495 & 172.29 & 357.02 & 2.5056 & 206.01 \\
\hline 343.25 & 1.9054 & 148.67 & 353.13 & 2.5216 & 223.60 & 355.24 & 2.2886 & 177.69 & 357.03 & 2.5368 & 211.33 \\
\hline 343.24 & 1.9225 & 151.01 & 353.13 & 2.5455 & 228.69 & 355.24 & 2.3246 & 182.73 & 357.03 & 2.5673 & 216.75 \\
\hline 343.22 & 1.9391 & 153.27 & 353.14 & 2.5669 & 233.65 & 355.24 & 2.3632 & 188.54 & 357.03 & 2.5958 & 221.98 \\
\hline 343.21 & 1.9556 & 155.71 & 353.13 & 2.5871 & 238.54 & 355.24 & 2.4181 & 197.16 & 357.04 & 2.6233 & 227.25 \\
\hline 343.18 & 1.9720 & 158.13 & 353.13 & 2.6065 & 243.48 & 355.24 & 2.4750 & 206.70 & 357.04 & 2.6502 & 232.68 \\
\hline 343.22 & 1.9832 & 159.75 & 353.13 & 2.6241 & 248.17 & 355.24 & 2.5285 & 216.42 & 357.03 & 2.6749 & 237.96 \\
\hline 343.24 & 1.9963 & 161.60 & 353.13 & 2.6411 & 252.95 & 355.24 & 2.5783 & 226.29 & 357.04 & 2.6927 & 241.91 \\
\hline 343.23 & 2.0108 & 163.82 & 353.13 & 2.6582 & 257.83 & 355.24 & 2.6212 & 235.50 & 357.04 & 2.7212 & 248.45 \\
\hline 343.25 & 2.0246 & 165.97 & 353.13 & 2.6734 & 262.52 & 355.24 & 2.6635 & 245.20 & 357.04 & 2.7567 & 257.14 \\
\hline 343.24 & 2.0379 & 168.09 & 353.13 & 2.6872 & 267.15 & 355.24 & 2.6996 & 254.54 & 357.04 & 2.7878 & 265.41 \\
\hline 343.23 & 2.0520 & 170.29 & 353.13 & 2.7007 & 271.80 & 355.25 & 2.7333 & 263.91 & 357.04 & 2.8115 & 272.21 \\
\hline 343.25 & 2.0658 & 172.70 & 353.13 & 2.7134 & 276.52 & 355.24 & 2.7628 & 273.22 & 357.04 & 2.8375 & 280.22 \\
\hline 343.25 & 2.0793 & 174.97 & 353.13 & 2.7249 & 281.02 & 355.24 & 2.7902 & 282.60 & 357.04 & 2.8621 & 288.49 \\
\hline 343.25 & 2.0927 & 177.24 & 353.13 & 2.7342 & 285.02 & 355.24 & 2.8116 & 290.74 & 357.03 & 2.8830 & 296.29 \\
\hline 343.25 & 2.1060 & 179.66 & 353.13 & 2.7444 & 289.45 & 355.24 & 2.8311 & 299.13 & 357.03 & 2.8951 & 301.32 \\
\hline
\end{tabular}




\begin{tabular}{|c|c|c|c|c|c|c|c|c|c|c|c|}
\hline 343.21 & 2.1185 & 181.99 & 353.12 & 2.7537 & 293.77 & 355.24 & 2.8486 & 307.39 & 357.03 & 2.9003 & 303.89 \\
\hline$T$ & $P$ & $\rho$ & $T$ & $P$ & $\rho$ & $T$ & $P$ & $\rho$ & $T$ & $P$ & $\rho$ \\
\hline $\mathrm{K}$ & $\mathrm{MPa}$ & $\mathrm{kg} \cdot \mathrm{m}^{-3}$ & $\mathrm{~K}$ & $\mathrm{MPa}$ & $\mathrm{kg} \cdot \mathrm{m}^{-3}$ & K & $\mathrm{MPa}$ & $\mathrm{kg} \cdot \mathrm{m}^{-3}$ & K & $\mathrm{MPa}$ & $\mathrm{kg} \cdot \mathrm{m}^{-3}$ \\
\hline 357.03 & 2.9107 & 308.24 & 358.11 & 2.3484 & 180.01 & 358.14 & 3.0297 & 350.44 & 362.88 & 2.5683 & 199.28 \\
\hline 357.03 & 2.9234 & 313.91 & 358.12 & 2.3729 & 183.30 & 358.16 & 3.0785 & 391.31 & 362.88 & 2.6231 & 207.21 \\
\hline 357.03 & 2.9363 & 320.08 & 358.12 & 2.3945 & 186.40 & & & & 362.87 & 2.6736 & 215.10 \\
\hline 357.03 & 2.9582 & 331.37 & 358.12 & 2.4147 & 189.24 & 362.89 & 1.6026 & 98.83 & 362.88 & 2.7182 & 222.25 \\
\hline 357.03 & 2.9777 & 343.50 & 358.12 & 2.4316 & 191.77 & 362.90 & 1.6666 & 104.27 & 362.88 & 2.7545 & 228.42 \\
\hline \multirow[t]{2}{*}{357.05} & 3.0070 & 365.19 & 358.13 & 2.4492 & 194.38 & 362.90 & 1.7208 & 108.65 & 362.87 & 2.7843 & 233.81 \\
\hline & & & 358.12 & 2.4637 & 196.64 & 362.90 & 1.7711 & 112.78 & 362.87 & 2.8064 & 237.98 \\
\hline 358.10 & 1.6004 & 101.77 & 358.12 & 2.4802 & 199.08 & 362.89 & 1.8181 & 116.83 & 362.87 & 2.8272 & 241.92 \\
\hline 358.10 & 1.6902 & 109.47 & 358.13 & 2.4961 & 201.53 & 362.89 & 1.8613 & 120.81 & 362.86 & 2.8487 & 246.13 \\
\hline 358.10 & 1.7717 & 116.73 & 358.12 & 2.5087 & 203.68 & 362.89 & 1.8920 & 123.41 & 362.87 & 2.8840 & 253.16 \\
\hline 358.10 & 1.8445 & 123.53 & 358.12 & 2.5211 & 205.69 & 362.89 & 1.9269 & 126.55 & 362.87 & 2.9014 & 256.91 \\
\hline 358.11 & 1.9099 & 129.77 & 358.13 & 2.5339 & 207.80 & 362.89 & 1.9594 & 129.59 & 362.87 & 2.9269 & 262.44 \\
\hline 358.10 & 1.9529 & 133.98 & 358.13 & 2.5466 & 209.81 & 362.89 & 1.9911 & 132.66 & 362.87 & 2.9783 & 274.33 \\
\hline 358.11 & 2.0055 & 139.40 & 358.12 & 2.5553 & 211.41 & 362.88 & 2.0213 & 135.65 & 362.88 & 3.0746 & 299.37 \\
\hline 358.11 & 2.0530 & 144.47 & 358.13 & 2.6325 & 225.14 & 362.88 & 2.0506 & 138.29 & 362.90 & 3.1534 & 325.03 \\
\hline 358.11 & 2.0992 & 149.52 & 358.13 & 2.7116 & 241.11 & 362.88 & 2.0762 & 141.04 & 362.89 & 3.2150 & 350.25 \\
\hline 358.10 & 2.1400 & 154.11 & 358.13 & 2.7772 & 256.23 & 362.88 & 2.1156 & 144.99 & 362.89 & 3.2634 & 375.24 \\
\hline 358.10 & 2.1760 & 158.32 & 358.13 & 2.8259 & 269.03 & 362.88 & 2.1828 & 152.15 & 362.89 & 3.3028 & 401.16 \\
\hline 358.10 & 2.2109 & 162.48 & 358.13 & 2.8632 & 279.95 & 362.88 & 2.2493 & 159.13 & 362.89 & 3.3321 & 426.48 \\
\hline 358.11 & 2.2430 & 166.31 & 358.13 & 2.8932 & 289.65 & 362.88 & 2.3136 & 166.43 & 362.90 & 3.3554 & 452.56 \\
\hline 358.11 & 2.2741 & 170.22 & 358.14 & 2.9456 & 308.34 & 362.88 & 2.3824 & 174.89 & 362.90 & 3.3735 & 478.83 \\
\hline 358.11 & 2.2994 & 173.49 & 358.13 & 2.9749 & 320.98 & 362.87 & 2.4454 & 182.79 & 362.90 & 3.3855 & 500.09 \\
\hline 358.11 & 2.3229 & 176.62 & 358.13 & 2.9952 & 330.70 & 362.88 & 2.5108 & 191.35 & & & \\
\hline
\end{tabular}

Liquid and supercritical densities

\begin{tabular}{cccccccccccc}
\hline$T$ & $P$ & $\rho$ & $T$ & $P$ & $\rho$ & $T$ & $P$ & $\rho$ & $T$ & $P$ & $\rho$ \\
\hline $\mathrm{K}$ & $\mathrm{MPa}$ & $\mathrm{kg} \cdot \mathrm{m}^{-3}$ & $\mathrm{~K}$ & $\mathrm{MPa}$ & $\mathrm{kg} \cdot \mathrm{m}^{-3}$ & $\mathrm{~K}$ & $\mathrm{MPa}$ & $\mathrm{kg} \cdot \mathrm{m}^{-3}$ & $\mathrm{~K}$ & $\mathrm{MPa}$ & $\mathrm{kg} \cdot \mathrm{m}^{-3}$ \\
\hline 263.49 & 0.2344 & 1462.60 & 263.49 & 3.3443 & 1477.99 & 263.50 & 6.8018 & 1493.30 & 283.17 & 0.5712 & 1382.89 \\
263.49 & 0.4005 & 1463.48 & 263.50 & 3.4480 & 1478.47 & 263.51 & 7.0484 & 1494.28 & 283.17 & 0.6658 & 1383.60 \\
263.49 & 0.5098 & 1464.06 & 263.50 & 3.5897 & 1479.11 & 263.50 & 7.2675 & 1495.21 & 283.17 & 0.7214 & 1383.94 \\
263.50 & 0.6902 & 1464.99 & 263.49 & 3.7777 & 1480.02 & 263.51 & 7.5076 & 1496.20 & 283.16 & 0.8365 & 1384.86 \\
263.50 & 0.7793 & 1465.45 & 263.50 & 3.9414 & 1480.72 & 263.49 & 7.7506 & 1497.24 & 283.16 & 1.0396 & 1386.34 \\
263.49 & 0.8440 & 1465.81 & 263.50 & 4.1241 & 1481.55 & 263.52 & 8.0413 & 1498.34 & 283.17 & 1.6324 & 1390.59 \\
263.50 & 1.1187 & 1467.16 & 263.51 & 4.3378 & 1482.55 & 263.51 & 8.3648 & 1499.70 & 283.16 & 2.2501 & 1394.87 \\
263.49 & 1.4771 & 1469.01 & 263.50 & 4.6165 & 1483.82 & 263.50 & 8.5930 & 1500.66 & 283.16 & 2.6648 & 1397.57 \\
263.49 & 1.8313 & 1470.78 & 263.50 & 4.9541 & 1485.33 & 263.50 & 8.8186 & 1501.54 & 283.15 & 2.7118 & 1397.93 \\
263.49 & 2.1807 & 1472.49 & 263.50 & 5.2620 & 1486.69 & 263.50 & 9.0842 & 1502.59 & 283.14 & 2.8054 & 1398.54 \\
263.50 & 2.5772 & 1474.41 & 263.50 & 5.6047 & 1488.17 & 263.50 & 9.3944 & 1503.80 & 283.16 & 2.9493 & 1399.47 \\
263.49 & 2.8130 & 1475.50 & 263.50 & 6.0257 & 1490.01 & 263.50 & 9.7162 & 1505.07 & 283.16 & 2.9927 & 1399.77 \\
263.49 & 2.9795 & 1476.28 & 263.49 & 6.2416 & 1490.94 & 263.51 & 9.99 & 1506.07 & 283.17 & 2.9971 & 1399.77 \\
263.49 & 3.1959 & 1477.28 & 263.50 & 6.4174 & 1491.65 & & & & 283.17 & 2.9998 & 1399.80
\end{tabular}




\begin{tabular}{|c|c|c|c|c|c|c|c|c|c|c|c|}
\hline 263.50 & 3.2614 & 1477.57 & 263.50 & 6.5911 & 1492.39 & 283.17 & 0.4698 & 1382.04 & 283.17 & 3.0382 & 1400.05 \\
\hline$T$ & $P$ & $\rho$ & $T$ & $P$ & $\rho$ & $T$ & $P$ & $\rho$ & $T$ & $P$ & $\rho$ \\
\hline K & $\mathrm{MPa}$ & $\mathrm{kg} \cdot \mathrm{m}^{-3}$ & K & $\mathrm{MPa}$ & $\mathrm{kg} \cdot \mathrm{m}^{-3}$ & K & $\mathrm{MPa}$ & $\mathrm{kg} \cdot \mathrm{m}^{-3}$ & K & $\mathrm{MPa}$ & $\mathrm{kg} \cdot \mathrm{m}^{-3}$ \\
\hline 283.16 & 3.1615 & 1400.87 & 303.37 & 2.7849 & 1309.16 & 323.36 & 3.2630 & 1209.83 & 343.18 & 3.2770 & 1072.74 \\
\hline 283.16 & 3.2296 & 1401.33 & 303.36 & 2.9185 & 1310.51 & 323.37 & 3.5588 & 1214.48 & 343.14 & 3.4333 & 1078.74 \\
\hline 283.16 & 3.2728 & 1401.61 & 303.36 & 2.9307 & 1310.67 & 323.36 & 3.6720 & 1216.27 & 343.15 & 3.5963 & 1084.50 \\
\hline 283.16 & 3.3637 & 1402.20 & 303.38 & 2.9933 & 1311.27 & 323.36 & 3.7893 & 1218.14 & 343.10 & 3.8060 & 1091.78 \\
\hline 283.17 & 3.5116 & 1403.13 & 303.37 & 3.0866 & 1312.17 & 323.36 & 3.9397 & 1220.38 & 343.10 & 3.9148 & 1095.09 \\
\hline 283.17 & 3.6663 & 1404.11 & 303.38 & 3.1857 & 1313.13 & 323.36 & 4.0934 & 1222.65 & 343.10 & 4.0523 & 1099.41 \\
\hline 283.15 & 3.8026 & 1405.02 & 303.37 & 3.2693 & 1313.88 & 323.36 & 4.2825 & 1225.41 & 343.15 & 4.1954 & 1103.09 \\
\hline 283.15 & 3.9872 & 1406.17 & 303.37 & 3.3126 & 1314.39 & 323.37 & 4.4794 & 1228.20 & 343.15 & 4.3449 & 1107.25 \\
\hline 283.15 & 4.1863 & 1407.41 & 303.37 & 3.4926 & 1316.15 & 323.36 & 4.6979 & 1231.27 & 343.08 & 4.4776 & 1111.27 \\
\hline 283.15 & 4.4758 & 1409.19 & 303.37 & 3.7235 & 1318.30 & 323.36 & 4.9172 & 1234.22 & 343.13 & 4.6043 & 1114.20 \\
\hline 283.16 & 4.8504 & 1411.46 & 303.37 & 3.9594 & 1320.48 & 323.36 & 5.1432 & 1237.21 & 343.14 & 4.7474 & 1117.85 \\
\hline 283.16 & 5.1823 & 1413.45 & 303.36 & 4.2842 & 1323.48 & 323.37 & 5.3859 & 1240.33 & 343.13 & 4.8853 & 1121.19 \\
\hline 283.16 & 5.4802 & 1415.20 & 303.37 & 4.7074 & 1327.25 & 323.36 & 5.6525 & 1243.74 & 343.14 & 5.0337 & 1124.78 \\
\hline 283.17 & 5.6908 & 1416.42 & 303.37 & 5.1888 & 1331.39 & 323.36 & 5.8633 & 1246.31 & 343.16 & 5.1610 & 1127.67 \\
\hline 283.17 & 5.9249 & 1417.79 & 303.37 & 5.5877 & 1334.67 & 323.36 & 6.0183 & 1248.17 & 343.11 & 5.2809 & 1130.51 \\
\hline 283.16 & 6.1474 & 1419.06 & 303.36 & 5.7419 & 1335.96 & 323.36 & 6.2000 & 1250.33 & 343.15 & 5.4296 & 1133.72 \\
\hline 283.17 & 6.4221 & 1420.61 & 303.38 & 5.9178 & 1337.36 & 323.37 & 6.3922 & 1252.57 & 343.13 & 5.5803 & 1136.99 \\
\hline 283.16 & 6.6614 & 1421.98 & 303.37 & 6.0895 & 1338.75 & 323.36 & 6.5607 & 1254.59 & 343.13 & 5.7458 & 1140.49 \\
\hline 283.16 & 6.9272 & 1423.44 & 303.36 & 6.2521 & 1340.07 & 323.37 & 6.7801 & 1257.08 & 343.09 & 5.9144 & 1144.10 \\
\hline 283.16 & 7.1758 & 1424.82 & 303.37 & 6.4774 & 1341.86 & 323.36 & 7.0081 & 1259.57 & 343.13 & 6.1494 & 1148.49 \\
\hline 283.16 & 7.4145 & 1426.14 & 303.37 & 6.7016 & 1343.59 & 323.37 & 7.2360 & 1262.04 & 343.15 & 6.3754 & 1152.64 \\
\hline 283.16 & 7.8586 & 1428.50 & 303.36 & 6.9381 & 1345.42 & 323.36 & 7.4970 & 1264.90 & 343.12 & 6.6420 & 1157.63 \\
\hline 283.16 & 8.3282 & 1431.01 & 303.37 & 7.2007 & 1347.40 & 323.37 & 7.7482 & 1267.53 & 343.12 & 6.9374 & 1162.77 \\
\hline 283.16 & 8.6936 & 1432.92 & 303.37 & 7.4843 & 1349.56 & 323.36 & 8.0400 & 1270.52 & 343.16 & 7.1456 & 1166.09 \\
\hline 283.16 & 9.1257 & 1435.16 & 303.37 & 7.7694 & 1351.63 & 323.37 & 8.3358 & 1273.49 & 343.13 & 7.3143 & 1169.11 \\
\hline 283.17 & 9.6012 & 1437.53 & 303.37 & 8.0990 & 1354.02 & 323.36 & 8.6540 & 1276.62 & 343.15 & 7.5136 & 1172.21 \\
\hline \multirow[t]{2}{*}{283.16} & 10.00 & 1439.52 & 303.38 & 8.4005 & 1356.17 & 323.36 & 8.8450 & 1278.47 & 343.15 & 7.7097 & 1175.33 \\
\hline & & & 303.38 & 8.7837 & 1358.84 & 323.36 & 9.0517 & 1280.45 & 343.13 & 7.9291 & 1178.74 \\
\hline 303.36 & 0.85 & 1287.97 & 303.38 & 9.1799 & 1361.62 & 323.36 & 9.2694 & 1282.50 & 343.13 & 8.1778 & 1182.49 \\
\hline 303.37 & 0.9378 & 1288.97 & 303.37 & 9.7063 & 1365.14 & 323.36 & 9.4714 & 1284.41 & 343.13 & 8.4071 & 1185.80 \\
\hline 303.37 & 0.9511 & 1289.14 & 303.37 & 9.99 & 1367.03 & 323.36 & 9.7097 & 1286.55 & 343.10 & 8.6707 & 1189.51 \\
\hline 303.36 & 1.0541 & 1290.38 & 323.37 & 1.42 & 1174.31 & 323.36 & 9.9458 & 1288.68 & 343.12 & 9.0310 & 1194.37 \\
\hline 303.37 & 1.1573 & 1291.56 & 323.36 & 1.5746 & 1177.86 & 323.36 & 9.99 & 1289.15 & 343.17 & 9.4439 & 1199.72 \\
\hline 303.36 & 1.2988 & 1293.20 & 323.36 & 1.7619 & 1181.93 & & & & 343.07 & 9.8832 & 1205.55 \\
\hline 303.37 & 1.4773 & 1295.32 & 323.36 & 1.9856 & 1186.54 & 343.11 & 2.38 & 1030.41 & 343.13 & 9.9823 & 1206.55 \\
\hline 303.36 & 1.6090 & 1296.75 & 323.37 & 2.1895 & 1190.59 & 343.18 & 2.5875 & 1041.95 & & & \\
\hline 303.36 & 1.8082 & 1298.96 & 323.36 & 2.4451 & 1195.49 & 343.18 & 2.7999 & 1052.25 & 348.15 & 2.5063 & 959.57 \\
\hline 303.36 & 1.9951 & 1301.01 & 323.36 & 2.7330 & 1200.72 & 343.17 & 2.8793 & 1056.54 & 348.15 & 2.5807 & 967.62 \\
\hline 303.37 & 2.2154 & 1303.37 & 323.36 & 2.8503 & 1202.76 & 343.17 & 2.9726 & 1060.25 & 348.15 & 2.6650 & 975.74 \\
\hline 303.36 & 2.4610 & 1305.98 & 323.36 & 2.9981 & 1205.34 & 343.14 & 3.0434 & 1063.62 & 348.15 & 2.7670 & 984.43 \\
\hline
\end{tabular}




\begin{tabular}{|c|c|c|c|c|c|c|c|c|c|c|c|}
\hline 303.37 & 2.6970 & 1308.28 & 323.36 & 3.0089 & 1205.47 & 343.18 & 3.1646 & 1068.25 & 348.14 & 2.8350 & 989.71 \\
\hline$T$ & $P$ & $\rho$ & $T$ & $P$ & $\rho$ & $T$ & $P$ & $\rho$ & $T$ & $P$ & $\rho$ \\
\hline K & $\mathrm{MPa}$ & $\mathrm{kg} \cdot \mathrm{m}^{-3}$ & $\mathrm{~K}$ & $\mathrm{MPa}$ & $\mathrm{kg} \cdot \mathrm{m}^{-3}$ & $\mathrm{~K}$ & $\mathrm{MPa}$ & $\mathrm{kg} \cdot \mathrm{m}^{-3}$ & K & $\mathrm{MPa}$ & $\mathrm{kg} \cdot \mathrm{m}^{-3}$ \\
\hline 348.15 & 2.8998 & 994.57 & 353.10 & 2.9255 & 912.33 & 357.02 & 3.1111 & 833.74 & 362.88 & 3.5137 & 727.62 \\
\hline 348.15 & 2.9771 & 999.82 & 353.12 & 2.9622 & 917.80 & 357.02 & 3.1712 & 852.76 & 362.90 & 3.5720 & 762.04 \\
\hline 348.15 & 2.9924 & 1000.80 & 353.12 & 2.9893 & 921.43 & 357.01 & 3.2529 & 871.60 & 362.92 & 3.6667 & 797.39 \\
\hline 348.13 & 3.0241 & 1003.27 & 353.13 & 2.9998 & 922.83 & 357.01 & 3.3072 & 882.00 & 362.92 & 3.7773 & 826.35 \\
\hline 348.15 & 3.1686 & 1011.94 & 353.13 & 3.0507 & 929.65 & 357.02 & 3.3780 & 893.55 & 362.93 & 3.8981 & 849.28 \\
\hline 348.14 & 3.3388 & 1021.49 & 353.10 & 3.1188 & 938.02 & 357.03 & 3.4703 & 906.34 & 362.92 & 3.9491 & 857.62 \\
\hline 348.13 & 3.5586 & 1032.40 & 353.13 & 3.2195 & 948.23 & 357.03 & 3.5628 & 917.41 & 362.92 & 4.0139 & 867.25 \\
\hline 348.14 & 3.6615 & 1037.05 & 353.14 & 3.3175 & 957.33 & 357.01 & 3.6121 & 923.16 & 362.92 & 4.0824 & 876.48 \\
\hline 348.14 & 3.7808 & 1042.19 & 353.13 & 3.4302 & 966.82 & 357.00 & 3.6702 & 929.31 & 362.92 & 4.1528 & 885.28 \\
\hline 348.15 & 3.8951 & 1046.96 & 353.13 & 3.5523 & 976.05 & 357.01 & 3.7558 & 937.42 & 362.91 & 4.2311 & 894.18 \\
\hline 348.14 & 4.0246 & 1052.08 & 353.13 & 3.6916 & 985.56 & 357.01 & 3.8510 & 945.66 & 362.91 & 4.3262 & 903.88 \\
\hline 348.13 & 4.1628 & 1057.39 & 353.12 & 3.8631 & 996.09 & 357.01 & 3.9570 & 954.08 & 362.91 & 4.4244 & 913.15 \\
\hline 348.13 & 4.3298 & 1063.41 & 353.13 & 4.0712 & 1007.44 & 357.02 & 4.0890 & 963.54 & 362.90 & 4.5292 & 922.20 \\
\hline 348.14 & 4.5222 & 1069.87 & 353.12 & 4.3084 & 1019.03 & 357.02 & 4.2311 & 972.86 & 362.89 & 4.6511 & 931.84 \\
\hline 348.14 & 4.7012 & 1075.55 & 353.12 & 4.5492 & 1029.60 & 357.02 & 4.4027 & 983.12 & 362.90 & 4.8021 & 942.47 \\
\hline 348.14 & 4.8352 & 1079.64 & 353.12 & 4.7878 & 1039.11 & 357.02 & 4.5908 & 993.37 & 362.92 & 4.9814 & 953.96 \\
\hline 348.13 & 4.9330 & 1082.55 & 353.13 & 5.0040 & 1047.06 & 357.02 & 4.8261 & 1004.87 & 362.92 & 5.1603 & 964.26 \\
\hline 348.13 & 5.0774 & 1086.71 & 353.13 & 5.2259 & 1054.73 & 357.02 & 5.0853 & 1016.35 & 362.92 & 5.2834 & 970.93 \\
\hline 348.14 & 5.2077 & 1090.33 & 353.13 & 5.4583 & 1062.16 & 357.02 & 5.3577 & 1027.14 & 362.93 & 5.4501 & 979.39 \\
\hline 348.14 & 5.3649 & 1094.65 & 353.13 & 5.6592 & 1068.32 & 357.03 & 5.5121 & 1032.88 & 362.92 & 5.5832 & 985.74 \\
\hline 348.14 & 5.5644 & 1099.78 & 353.13 & 5.8803 & 1074.71 & 357.02 & 5.6709 & 1038.54 & 362.93 & 5.7812 & 994.50 \\
\hline 348.13 & 5.7912 & 1105.44 & 353.13 & 6.0511 & 1079.42 & 357.02 & 5.8365 & 1044.18 & 362.93 & 5.9198 & 1000.30 \\
\hline 348.14 & 6.0365 & 1111.21 & 353.14 & 6.2400 & 1084.40 & 357.02 & 6.0264 & 1050.30 & 362.92 & 6.0494 & 1005.54 \\
\hline 348.13 & 6.3062 & 1117.33 & 353.13 & 6.4190 & 1089.01 & 357.02 & 6.1898 & 1055.34 & 362.92 & 6.2176 & 1012.03 \\
\hline 348.14 & 6.6047 & 1123.73 & 353.15 & 6.6542 & 1094.70 & 357.00 & 6.3067 & 1058.93 & 362.92 & 6.3845 & 1018.10 \\
\hline 348.13 & 6.8303 & 1128.36 & 353.14 & 6.8350 & 1099.00 & 357.02 & 6.4757 & 1063.71 & 362.92 & 6.5749 & 1024.73 \\
\hline 348.12 & 7.0392 & 1132.55 & 353.14 & 7.0278 & 1103.39 & 357.01 & 6.6403 & 1068.34 & 362.91 & 6.7692 & 1031.15 \\
\hline 348.12 & 7.2462 & 1136.50 & 353.15 & 7.2508 & 1108.26 & 357.02 & 6.8284 & 1073.32 & 362.91 & 6.9763 & 1037.63 \\
\hline 348.12 & 7.4780 & 1140.85 & 353.14 & 7.4607 & 1112.69 & 357.02 & 7.0299 & 1078.51 & 362.91 & 7.2208 & 1044.90 \\
\hline 348.11 & 7.7121 & 1145.16 & 353.14 & 7.6879 & 1117.39 & 357.01 & 7.2375 & 1083.67 & 362.90 & 7.4626 & 1051.74 \\
\hline 348.12 & 7.9409 & 1149.12 & 353.13 & 7.9294 & 1122.16 & 357.02 & 7.4729 & 1089.23 & 362.89 & 7.7376 & 1059.06 \\
\hline 348.12 & 8.1996 & 1153.54 & 353.12 & 8.1999 & 1127.34 & 357.01 & 7.7275 & 1095.06 & 362.89 & 8.0418 & 1066.74 \\
\hline 348.12 & 8.4235 & 1157.17 & 353.09 & 8.4395 & 1131.92 & 357.01 & 7.9973 & 1100.94 & 362.89 & 8.3779 & 1074.76 \\
\hline 348.12 & 8.6747 & 1161.26 & 353.06 & 8.6677 & 1136.08 & 357.01 & 8.2949 & 1107.17 & 362.89 & 8.7464 & 1083.00 \\
\hline 348.13 & 8.9460 & 1165.39 & 353.06 & 9.0076 & 1141.95 & 357.02 & 8.6373 & 1113.95 & 362.89 & 8.9576 & 1087.48 \\
\hline 348.14 & 9.2995 & 1170.72 & 353.07 & 9.3594 & 1147.74 & 357.02 & 8.9868 & 1120.56 & 362.88 & 9.1065 & 1090.63 \\
\hline 348.16 & 9.95 & 1179.71 & 353.07 & 9.7590 & 1154.06 & 357.01 & 9.3930 & 1127.88 & 362.88 & 9.2777 & 1094.12 \\
\hline 353.13 & 2.79 & 883.82 & 353.06 & 10.00 & 1157.73 & 357.02 & 9.8823 & 1136.21 & 362.88 & 9.4571 & 1097.66 \\
\hline 353.12 & 2.8200 & 892.36 & & & & 357.02 & 9.9954 & 1138.05 & 362.88 & 9.6459 & 1101.34 \\
\hline 353.10 & 2.8439 & 897.65 & 357.00 & 3.02 & 777.77 & & & & 362.88 & 9.8470 & 1105.11 \\
\hline
\end{tabular}


$\begin{array}{llllll}353.11 & 2.8887 & 906.09 & 357.02 & 3.0532 & 807.50\end{array}$

and 3 of original paper [3]. Other trustable data (about 10500 over the 48000 original raw data) are available from corresponding author: laugier@enscbp.fr.

\section{Conclusions}

Through the present study we have pointed out the importance of reliable and accurate data and the usefulness of simple data consistency tests. These simple tests justify the development of various performing techniques such as those based on neural network.

Density of gases and gas mixtures must tend to zero when pressure tends to zero and additionally the virial law must be followed.

Neural network based approach presently used to assess the quality of experimental hexafluoropropylene densities gives encouraging results.

For supercritical HFP only one isotherm has been conserved from previous work [3]. Due to high thermal effects in the vicinity of critical point, it would have been certainly necessary to be more careful and more patient recording the data (very small pressure changes are requested as a function of time).

Finally, we conclude neural network modeling is worth in the assessment of data consistency.

As far as high pressure vibrating tube densimeters are used, vapor densities at very low pressures are better determined through extrapolation of higher pressure values using virial equation of state.

Only trustable densities for the three vapor, liquid and supercritical physical states have been reported herein.

\section{Supporting Information Available:}

Density values of HFP at various temperatures and in its various states: vapor, liquid and supercritical. This material is available upon demand to corresponding author: laugier@enscbp.fr. It has to be used preferably to the material presented in Coquelet et al.'s paper [3] that unfortunately contains inaccurate and erroneous data.

\section{References}

[1] H. Madani, A. Valtz, C. Coquelet, A. H. Meniai and D. Richon, “(Vapor plus Liquid) Equilibrium Data for (Carbon Dioxide + 1,1-Difluoroethane) System at Temperatures from 258 to $343 \mathrm{~K}$ and Pressures up to about 8 MPa," The Journal of Chemical Thermodynamics, Vol.

\begin{tabular}{llllll}
362.88 & 3.47 & 691.30 & 362.88 & 9.9964 & 1107.95 \\
\hline
\end{tabular}

40, No. 10, 2008, pp. 1490-1494.

doi:10.1016/j.jct.2008.06.002

[2] G. Acerboni, J. A. Buekes, N. R. Jensen, J. Hjorth, G. Myhre, C. J. Nielsen and J. K. Sundet, “Atmospheric Degradation and Global Warming Potentials of Three Perfluoroalkenes," Atmospheric Environment, Vol. 35, No. 24, 2001, pp. 4113-4123. doi:10.1016/S1352-2310(01)00209-6

[3] C. Coquelet, D. Ramjugernath, H. Madani, A. Valtz, P. Naidoo and A. H. Meniai, "Experimental Measurements of Vapor Pressures and Densities of Pure Hexafluoropropylene,” Journal of Chemical \& Engineering Data, Vol. 55, No. 6, 2010, pp. 2093-2099. doi:10.1021/je900596d

[4] D. Richon, "Experimental Techniques for the Determination of Thermophysical Properties to Enhance Chemical Processes,” Pure and Applied Chemistry, Vol. 81, No. 10, 2009, pp. 1769-1782. doi:10.1351/PAC-CON-08-09-06

[5] C. Coquelet, L. A. Galicia-Luna, A. H. Mohammadi and D. Richon, "The Essential Importance of Experimental Research and the Use of Experimental Thermodynamics to the Benefit of Industry," Fluid Phase Equilibria, Vol. 296, No. 1, 2010, pp. 2-3.

doi:10.1016/j.fluid.2010.04.004

[6] D. Richon, 2009. http://www.wix.com/drichon/thermoadvices

[7] A. Eslamimanesh, A. H. Mohammadi and D. Richon, "Thermodynamic Consistency Test for Experimental Data of Water Content of Methane," AIChE Journal, Vol. 57, No. 9, 2011, pp. 2566-2573. doi:10.1002/aic.12462

[8] A. Eslamimanesh, M. Yazdizadeh, A. H. Mohammadi and D. Richon, "Experimental Data Assessment Test for Diamondoids Solubility in Gaseous System," Journal of Chemical \& Engineering Data, Vol. 56, No. 5, 2011, pp. 2655-2659. doi:10.1021/je200193n

[9] A. Eslamimanesh, A. H. Mohammadi and D. Richon, "Thermodynamic Consistency Test for Experimental Data of Sulfur Content of Hydrogen Sulfide," Journal of Chemical \& Engineering Data, Vol. 50, No. 6, 2011, pp. 3555-3563. doi:10.1021/ie1017332

[10] A. Chouai, S. Laugier and D. Richon, "Modeling of Thermodynamic Properties Using Neural NetworksApplication to Refrigerants,” Fluid Phase Equilibria, Vol. 199, No. 1-2, 2002, pp. 53-62. doi:10.1016/S0378-3812(01)00801-9

[11] S. Laugier, F. Rivolet and D. Richon, "New Volume Translation for Cubic Equations of State," Fluid Phase Equilibria, Vol. 259, No. 1, 2007, pp. 99-104. doi:10.1016/j.fluid.2007.04.032 